\title{
Low Temperature physics Conference
}

\author{
FREUDENSTADT, FED. REP. GERMANY, 6-8 APRIL 1972
}

W. Klose

The Low Temperature Physics Division of EPS held their first Conference in Freudenstadt (Black Forest, Fed. Rep. Germany) on 6-8 April 1972. Altogether 230 participants were registered from Austria, Denmark, Fed. Rep. Germany, Finland, France, Hungary, Israel, Italy, Lebanon, The $\mathrm{Ne}$ therlands, Norway, Poland, Portugal, Sweden, Switzerland, UK, USA, USSR, Yugoslavia.

Each day was devoted to one topic: Physics below $0.1 \mathrm{~K}$; Helium problems; Phase transitions and coexistence of phases.

Originally there were no parallel sessions planned and each morning and afternoon started with invited papers :

\section{Physics below O.IK}

O.V. Lounasmaa (Finland)

New methods for approaching absolute zero.

K.W. Taconis (The Netherlands)

The physics of ${ }^{3} \mathrm{He}-{ }^{4} \mathrm{He}$ refrigeration.

M. Kalvius (Fed. Rep. Germany)

Mössbauer spectroscopy at very low temperatures.

K. Andres (USA)

Hyperfine enhanced nuclear cooling and nuclear ordering in the millidegree Kelvin range.

E. Bucher (USA)

The physics of Van Vleck paramagnetic materials in view of their nuclear cooling properties.

Helium problems

D.V. Osborne (UK)

Evaporation and condensation of liquid $\mathrm{He}-\mathrm{Il}$

R. de Bruyn Ouboter (The Netherlands)

The hydrodynamics of the He film. J. Wheatley (USA)

Anomalously low thermal resistance between pure $\mathrm{He}^{3}$ and Curielaw paramagnetic substrates at very low temperatures.
Phase transitions and the coexistence of phases

J.E. Mercereau (USA)

Superconductivity at finite voltage.

\section{J. Zittartz (Fed. Rep. Germany)}

Magnetic impurities in superconductors.

P.C. Hohenberg (USA)

Magnetism in one and two dimensions.

Owing to the qualification of postdeadline papers, the original policy was changed and two short parallel sessions were allowed. Thus, about 50 contributed papers were presented at the Conference. A prize of DM 200. - was awarded to P. Leiderer (Physics Department, TH Munich) for the best standard in presenting his paper.

Scientific progress could be seen in all fields. K.W. Taconis reported on a new dilution refrigerator that achieved $50 \mathrm{mK}$ in a first run. K. Andres and $E$. Bucher opened up new perspectives to approaching absolute zero by Van Vleck type materials using hyperfine enhanced nuclear cooling, whereas O.V. Lounasmaa had pointed out the possibilities resting in Pome- ranchuk cooling. D.V. Osborne called attention to surface problems in $\mathrm{He}-\mathrm{II}$. R. de Bruyn Ouboter showed ingenious experiments on film properties of He-II. J. Zittartz announced the effect of Kondo-influenced superconductivity such that, below a certain lower transition temperature, superconductivity would vanish; that was confirmed experimentally by $\mathrm{G}$. von Minnigerode, G. Riblet and K. Winzer.

J. Wheatley, J.E. Mercereau and P.C. Hohenberg initiated interesting discussions and their contributions proposed a very solid ground for further research in the different fields. A most instructive film on $\mathrm{He}-\mathrm{Il}$ properties had been prepared and was presented by J.F. Allen.

Session-chairmen from 7 countries helped to run the Conference smoothly. The Conference Chairman takes this opportunity to thank all those who gave financial help to the Conference, namely: the Bundesministerium für Bildung und Wissenschaft, Bonn; Siemens AG, München-Erlangen; Linde AG, München; Philips Deutschland; BBC, Mannheim; AEGTelefunken, Frankfurt; Krupp, Essen; Messer-Griesheim, Frankfurt; RCA, Zürich; and Balzers, Balzers.

\section{The Impact of Computers on Physics}

\author{
CERN, Geneva, Switzerland, 10-14 April 1972
}

\section{Organized by the Computational Physics Group of EPS}

It was appropriate that this first European Conference on Computational Physics should have taken place at CERN with its 466 million Swiss Franc budget devoted to investigating the basic structure of matter by 'impact'. The Data Handling Division at CERN was engaged, during the Conference, in testing their newly installed CDC 7600 - the most power- ful computer for physics research in Europe.

The major conclusions of the successful Conference seemed to be that it was the initial formulation of phys- 